\title{
Augmented Coliseum: An Augmented Game Environment with Small Vehicles
}

\author{
Minoru KOJIMA*1, Maki SUGIMOTO*1 ${ }^{*}$, Akihiro NAKAMURA*1, \\ Masahiro TOMITA $^{* 2}$, Hideaki NII ${ }^{* 1}$ and Masahiko INAMI ${ }^{* 2 * 3}$ \\ ${ }^{*}$ Graduate School of The University of Electro-Communications \\ \{kojima,sugimoto,nakamura,nii\}@hi.mce.uec.ac.jp \\ ${ }^{* 2}$ Department of Mechanical Engineering Intelligent systems, \\ The University of Electro-Communications \\ \{tomita,inami\}@hi.mce.uec.ac.jp \\ *3Japan Science and Technology Agency, PRESTO
}

\begin{abstract}
We propose a novel display-based game environment using augmented reality technology with small robots. In this environment, the small robots can be augmented by a display image according to their positions and postures. The augmentation activity reinforces the fun of playing with such small robots in the real world.
\end{abstract}

\section{Introduction}

In this study, a game environment using augmented reality $(A R)$ technology that enables virtual functions for playing a game using small robots is proposed. Similar to how children use their imaginations to make a normal room into a battle field for playing games with toy tanks, we propose a way to virtually embody such imagination by superimposing computer graphics onto toys in the real world.

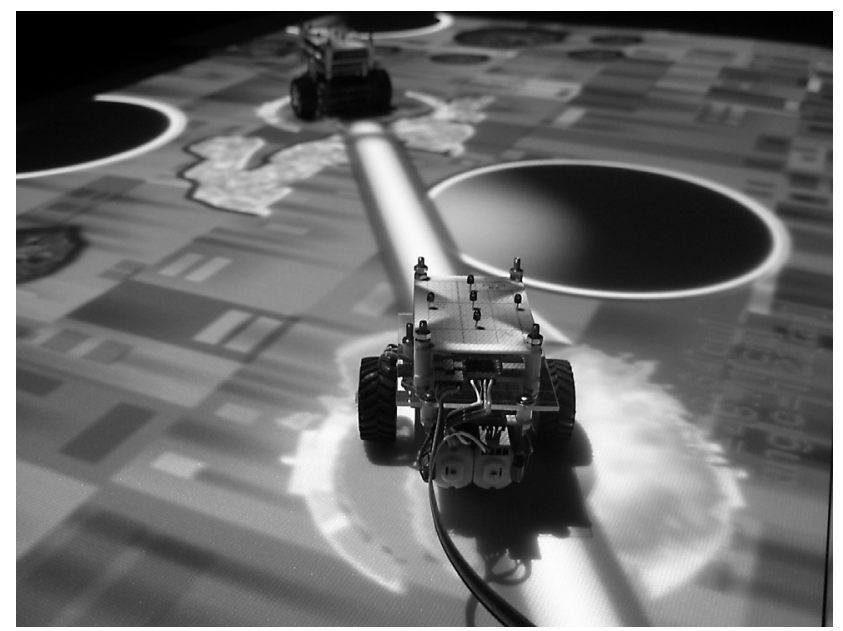

Figure 1. Game environment using AR with small vehicles.
Figure 1 shows a snapshot of such a game environment. Playing with toys such as small robots and vehicles is improved and enhanced by projecting images to the areas corresponding to the actual positions and directions of the toys. In addition, objects are able to express the phenomena of this augmented reality world by controlling actuators according to the results of simulations.

In the environment that we propose, the games are played with small robots and vehicles that can be treated as actual toys in the real world.

\section{Related Work}

$\mathrm{AR}$ is a technology that aims to enhance the real world by superimposing information onto it. Examples of this technology in entertainment environments include video see through-based systems that use head-mounted displays and cameras. Of such systems, the ARToolKit [1] is a significant tool that can be used to recognize items in the real world.

A real world shooting game environment combining augmented reality technology with wearable computing technology has been investigated [2], and there has been an attempt to create a game environment that realizes interaction with virtual objects using an over-head projector, physical interfaces, and embedded RFID tags. Moreover, in the field known as mixed reality, a study has reported the use of a projector to project the results of a simulation based on real clay geographical models using a laser range finder with an over-head projector [3]. Further, in a study that attempted to combine mixed reality and sports, an augmented ping-pong table using over-head projection that displayed ripples corresponding to the ball's movement [4] was created. In addition, Dynamic Shader Lamps [5] augment the surface of real objects with a projector. In other research areas involving mixed reality 
technologies, there are some reports of research using toys. One such study investigated human interfaces using block-type toy devices for the input of information [5]. Some other studies have used teddy bear-type Robotic User Interfaces incorporating telecommunication technology to synchronize the robot's posture [6] and to serve as a haptic interface for game environments [7].

\section{System Outline}

In our proposed system, there are two major areas of focus. One is the architecture of the augmented game system, and the other is a display-based measurement system.

\subsection{Augmented Game Environment}

Figure 2 shows the system outline of the environment.

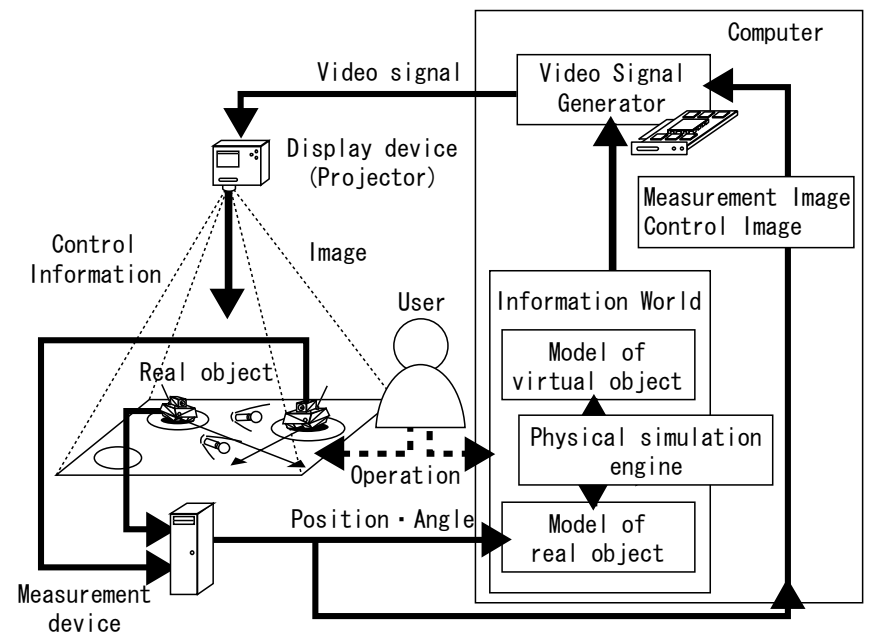

Figure 2. System outline
The main parts of this system are objects that have been enhanced by AR technology, the display device, and the information processing unit. In the system, there are models of the actual objects in the information world that are synchronized with the real world.

The interactions between the models of the actual objects and the virtual objects, which do not exist in the real world, are realized by physical simulation. For example, this physical simulation realizes collision detections using models of the real objects and virtual objects in the information world. Hence, interactions between the information and the real worlds can be achieved. Further, the physical simulation provides both reality and the rules for the information world. A user can move both the objects in the real world and the models of the objects in the information world via direct contact and controllers. In the system outline shown in Figure 2, the models of the objects are synchronized with the real objects by two methods; one is to measure the position and direction of the objects, and the other is to control the real objects through the information world. Figure 3 shows the sequence of this synchronization. If a real object is moved, then the model of that object can be modified by the information available from the measurement system. On the other hand, if the model of the object is moved as a result of the simulation, then the object can be moved by the control information from the information world. Hence, interactions between the information and the real worlds can be achieved.

This environment has the following features.

- The augmentation necessary for playing games is given to objects in the real world by the projected images.

- A large number of users and observers can experience games at same time because of the

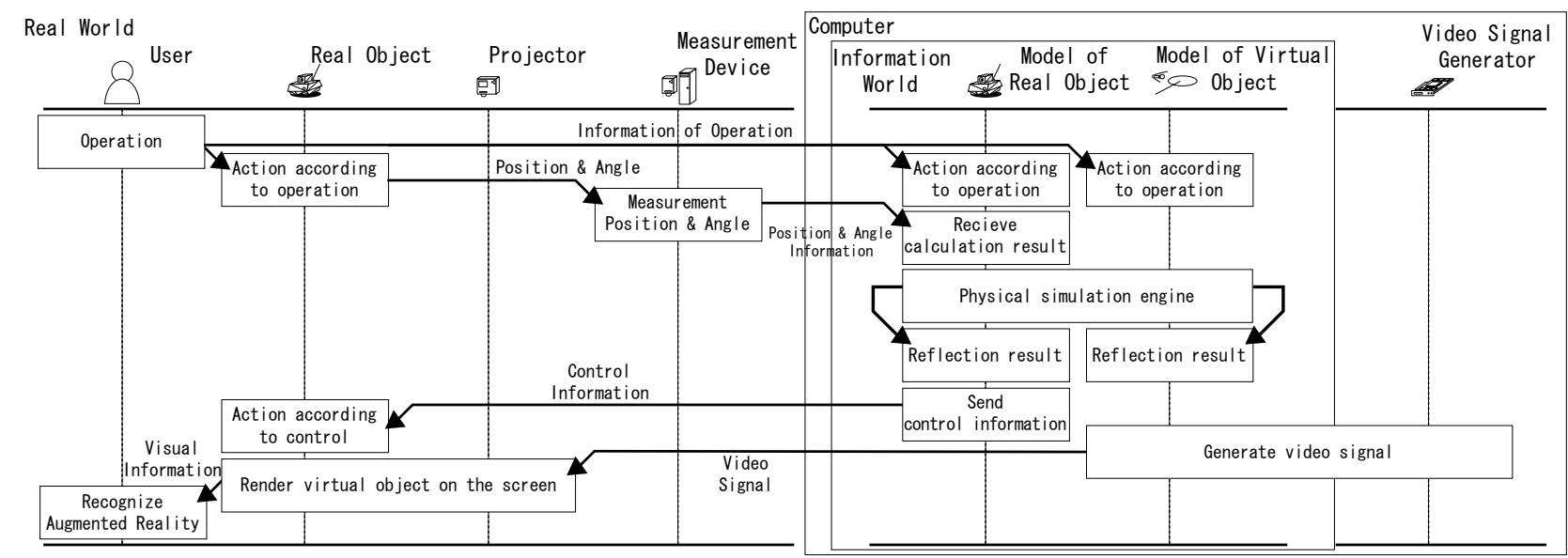

Figure 3. Synchronization sequence 
augmented reality environment using real objects with projected images.

- Users can interact physically with the real objects

- Real objects can interact with other real objects in the real world.

- Real objects can interact with virtual objects by making models of the objects in the information world.

- Real objects can represent accidents such as collisions in the information world using actuators.

\subsection{Display-Based Measurement System}

The display-based measurement system is a 2 dimensional tracking system using a display device equipped with a few brightness sensors. In an augmented reality environment based on a display device such as a projector, registrations between the real and the information worlds are necessary. Therefore, results of a display-based measurement system depend on fiducial images of the display, and the coordinate system for the measurements is the same as that of the output image displayed in the real world. Thus, a display-based measurement system suits the proposed system. Such a measurement system is used in this game environment to acquire the positions and directions of the objects. The display device draws the fiducial marker onto the real world. The sensors on the objects receive the fiducial marker, and the outputs of the sensors indicate the relative coordinates of the fiducial marker and the objects. Figure 4 shows a snap shot of robots tracked by this position and orientation measurement system.

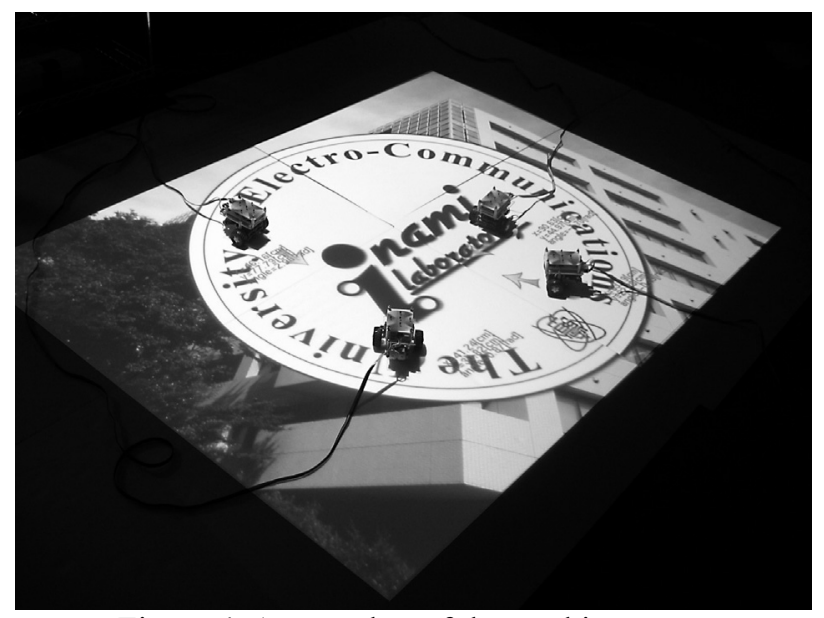

Figure 4. A snap shot of the tracking system

\subsubsection{The Principle of The Measurement.}

Figure 5 shows the fiducial marker actually used in Figure 4. The points in the Figure indicate the photo sensor layout. This fiducial marker enables measurement of the position and orientation of the robot. Expressions (1), (2), and (3) set out how to calculate the relative coordination. In this expression, $a^{\prime}{ }_{1}, a_{2}{ }_{2}, a^{\prime}{ }_{4}$, and $a^{\prime}{ }_{5}$ are the values in which $a_{3}$ has been subtracted from the output of sensors $a_{1}, a_{2}, a_{4}$, and $a_{5}$. The purpose of this process is to reduce the influence of ambient light as much as possible.

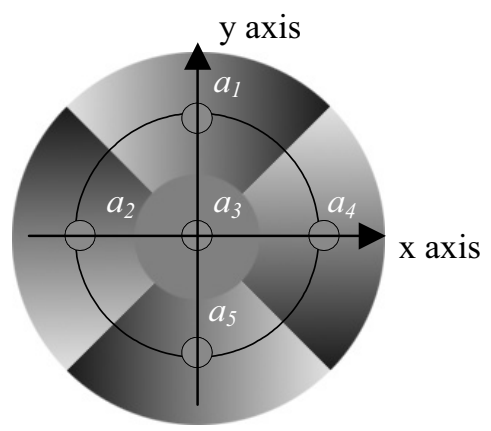

Figure 5. A fiducial marker to measure position and orientation

$$
\begin{aligned}
& a_{1}^{\prime}=a_{1}-a_{3} \\
& a_{2}^{\prime}=a_{2}-a_{3} \\
& a_{4}^{\prime}=a_{4}-a_{3} \\
& a_{5}^{\prime}=a_{5}-a_{3}
\end{aligned}
$$

(1) $d x \propto a_{1}^{\prime}-a_{5}^{\prime}$

(2) $d y \propto a_{2}^{\prime}-a_{4}^{\prime}$

(3) $\sin d \theta \propto a_{1}^{\prime}+a_{2}^{\prime}+a_{4}^{\prime}+a_{5}^{\prime}$

Figure 6 shows a fiducial marker used for obtaining the absolute orientation, which is calculated as shown by expressions (4), (5), and (6). In this expression, $a_{i}$ is the value of each sensor. $x_{i}$ and $y_{i}$ are the $x$ and $y$ coordinates of each sensor.

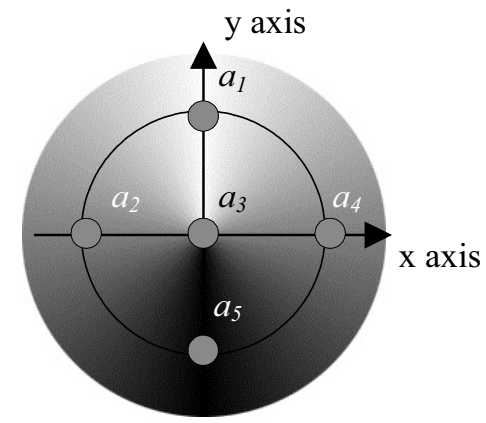

Figure 6. A fiducial marker to measure absolute orientation 
(4) $x=\frac{\sum_{i=1}^{n}\left(a_{i} \times x_{i}\right)}{\sum_{i=1}^{n} a_{i}}$
(5) $y=\frac{\sum_{i=1}^{n}\left(a_{i} \times y_{i}\right)}{\sum_{i=1}^{n} a_{i}}$

(6) $\theta=\arg (x+y j)$

\subsubsection{The Process of Tracking}

Regarding the sequence of the measurement system; first the system obtains the absolute positions of the objects by spatial division, as shown in Figure 7. For this, the system projects white on half domains of the screen. If the sensor recognizes white, there is a sensor somewhere in the white region. If the sensor recognizes black, there is a sensor somewhere in the black region. The system then narrows down the position of a sensor and so can measure an absolute position. Next, using the absolute orientation fiducial marker, the system acquires the orientations of the objects by calculating their center of gravity. After absolute detection, the system is able to track objects using the fiducial marker shown in Figure 5. However, in the present system it is not possible to search automatically for objects when the fiducial marker missed a object because of some problem. In such instances, the user must demand absolute position and orientation.

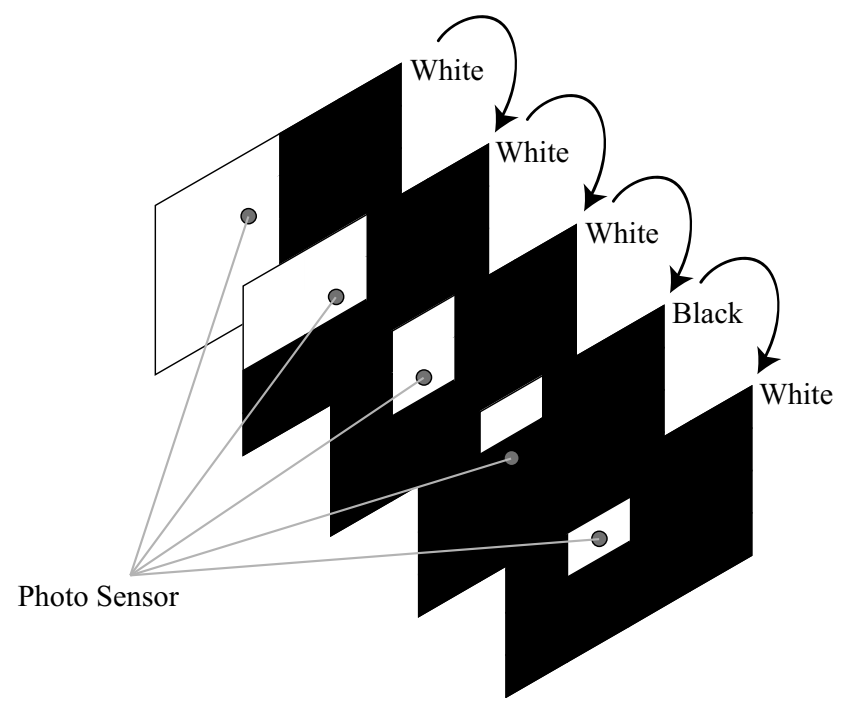

Figure 7. A search of absolute positions of object by spatial division
In a present system, there is a problem that the projected fiducial marker becomes an obstruction to augmenting the surfaces of the robots themselves. For instance, when a display panel such as an LCD is used, it will not become an obstruction because the fiducial marker is under the robots. Moreover, if infrared rays rather than visible light can be used for projection of the fiducial marker, this problem can be resolved.

We undertook a simple experiment in respect to the accuracy of the measurements by the fiducial marker of the measured position and orientation. For the experimental environment for the measurement, an overhead projector was mounted on the ceiling. The height difference of the projector to the sensors was $2.3(\mathrm{~m})$, and the screen size was $1430 \times 1080(\mathrm{~mm})$ at the plane on the sensors. The receiver units rested on the floor in a fixed position. The results of this experiment based on 5,000 samples; the standard deviation of $\mathrm{x}$ was $0.15(\mathrm{~mm})$, and $\mathrm{y}$ $0.13(\mathrm{~mm})$, and the angle was $0.39(\mathrm{deg})$.

A display-based measurement technique can be achieved by adding some photo sensors to the projection area. Thus, this technology is very effective for exhibition of installations and interactive techniques, etc.

\section{Experimental System}

The experimental system used in this study is composed of the following two parts.

- Small robots

- An augmented game environment

Both of these parts include units of the display-based measurement system. In this section, the actual implementations of each part are described.

\subsection{Small Robots}

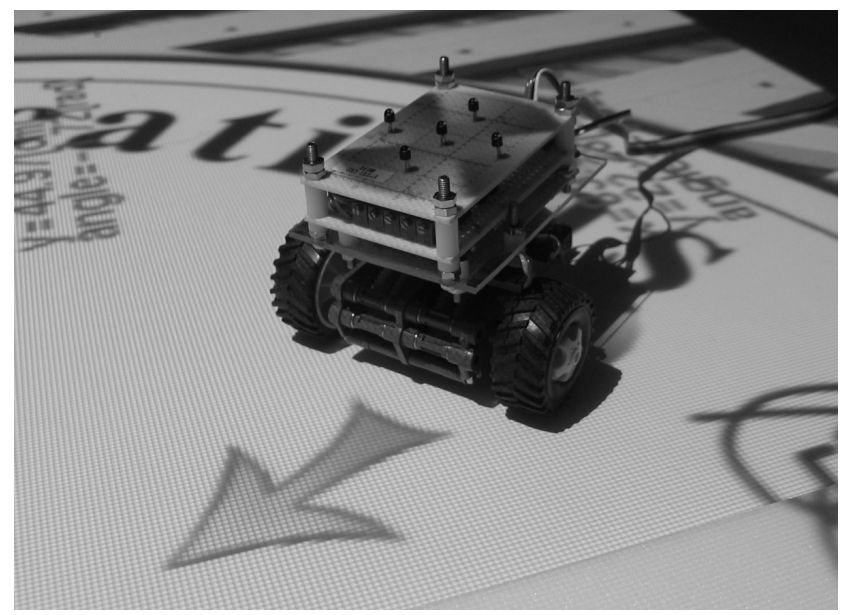

Figure 8. Small robot in the experimental system 
Figure 8 shows a small robot that has been augmented in this experimental system. The arrow projected in front of the robot displays the posture of the robot.

This small robot consists of a measurement device, which obtains the brightness projected from the display device, and a control device, which moves the robot using two motors and drivers. Figure 9 shows a block diagram of the small robot.

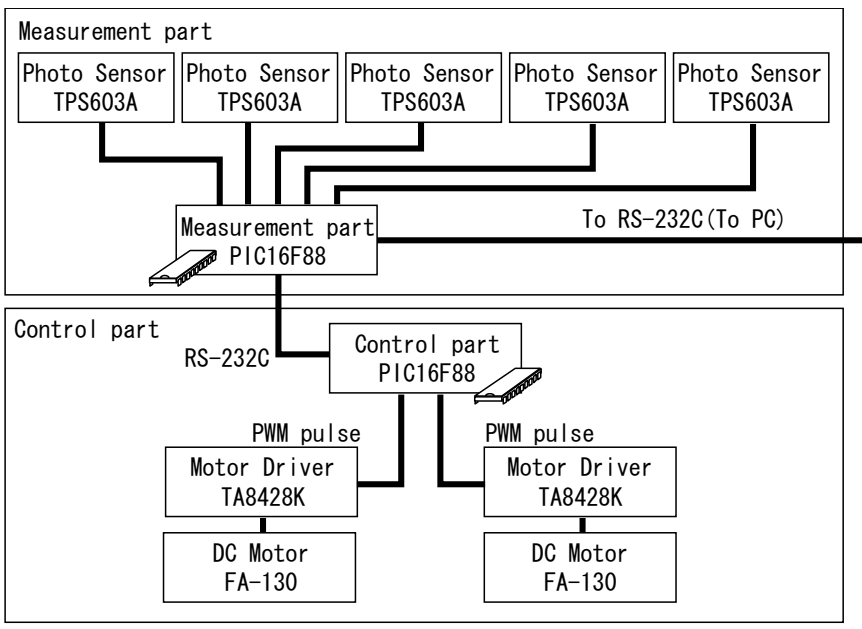

Figure 9. Block diagram of the small robot

A Micro Controller Unit (MCU) using a Microchip, PIC16F88, is used in both measurement and control units. The MCU of the measurement unit transmits brightness information to a PC that controls the whole system and receives control information from the robot and forwards it to the MCU of the control unit via the MCU of the measurement unit. There are also five photo transistors (TOSHIBA TPS 603A) in the measurement unit for obtaining fiducial marker information. Further, to control position and direction of the robot, two motor drivers (TOSHIBA TA8440H) are used in the control unit. The measurement MCU also transmits commands to the control MCU; thus, the control unit can be controlled by the measurement unit according to the fiducial marker.

\subsection{Augmented Game Environment}

In an experimental implementation of the augmented game environment, the devices shown in Table 1 were used.

\begin{tabular}{ll}
\hline Projector & NEC VT770 \\
CPU & AMD Athlon 64 3400+ \\
Memory & 1024MB \\
VideoCard & RADEON 9700 \\
OS & Windows XP Professional \\
\hline
\end{tabular}

Table 1. Devices
OpenGL and GLUT were used as resources for drawing computer graphics. To enable a fighting game in the environment using augmented reality technology, the small robots were decorated with computer graphics corresponding to their positions and directions.

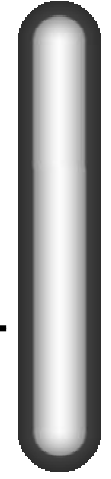

(a)

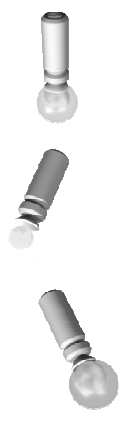

(b)

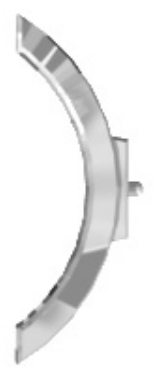

(c)

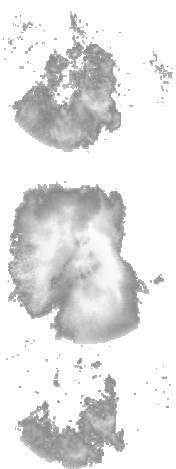

(d)
Figure 10. Examples of decoration images

Figure 10 shows images used to express the virtual functions of the objects in the game. Figure 10 (a) is a laser cannon, (b) is a missile, (c) is a shield, and (d) is used to express an explosion. In the program, these images are drawn as texture images on the polygons.

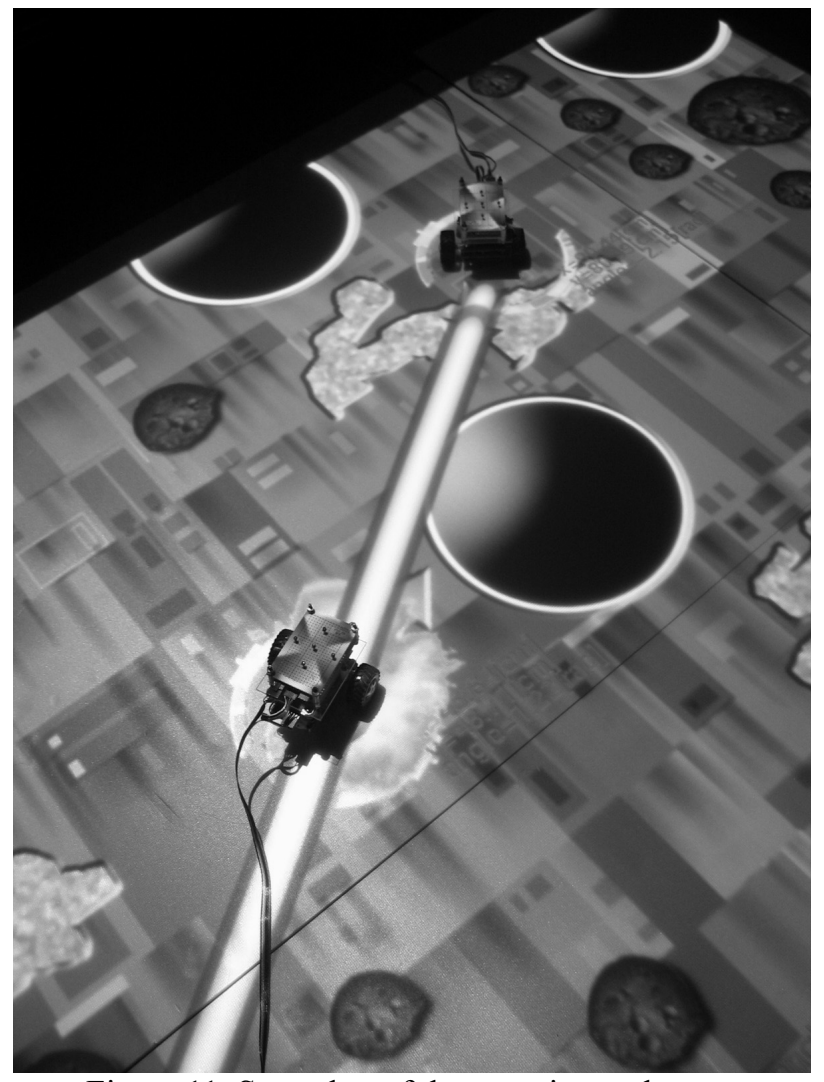

Figure 11. Snap shot of the experimental system 
Further, in this experimental system, the environment has a collision detection engine based on a circle that realizes interactions between models of real and virtual objects.

Figure 11 shows a scene in the augmented game environment. In this scene, a robot used a laser cannon to attack the other robot, and the bombarded robot has exploded. In the real environment, these robots only have their physically implemented functions. However, in the augmented game environment, these robots could be given virtual functions utilizing the computer graphics to play games.

\section{Conclusion}

In this study an augmented game environment using a display device and small robots was achieved. The environment was able to give virtual functions to robots in the real world to enable games to be played. Such an augmentation using AR technology can improve the entertainment value of commercialized toys and small robots by adding simple sensors.

In addition, we can use not only a projector but also other display devices for the display-based measurement system. For example, this system can be applied to a table-top system with rear-projection display, or to an LCD display.

\section{References}

[1] Hirokazu Kato, Mark Billinghurst, Ivan Poupyrev, Kenji Imamoto, Keihachiro Tachibana:Virtual Object Manipulation on a Table-Top AR Environment, Proc. of IEEE and ACM International Symposium on Augmented Reality 2000, pp.111-119 (2000)

[2] Bruce H. Thomas and Wayne Piekarski. Making augmented reality outdoor games:why is it hard? In In Production Process of 3D Computer Graphics Applications - Structures, Roles and Tools. ACM SIGGRAPH and Eurographics Campfire, Snowbird, Utah, USA, June 2002.

[3] Ben Piper, Carlo Ratti and Hiroshi Ishii, Illuminating Clay: A 3-D Tangible Interface for Landscape Analysis, Proceedings of Conference on Human Factors in Computing Systems 2002, pp. 355-362, 2002.

[4] Ishii, H., Wisneski, C., Orbanes, J., Chun, B., \& Paradiso, J., PingPongPlus: Design of an Athletic-Tangible Interface for Computer-Supported Cooperative Play. In proceedings of CHI '99, pp. 394-401, 1999

[5] Bandyopadhyay, D.; Raskar, R.; Fuchs, H. "Dynamic shader lamps: Painting on movable objects", IEEE and ACM International Symposium on Augmented Reality, 2001, pp. 207-216)

[6] Yoshifumi Kitamura, Yuichi Itoh, Toshihiro Masaki, Fumio Kishino, ActiveCube: A Bi-directional User Interface using Cubes, KES 2000: Fourth International Conference on Knowledge-Based Intelligent Engineering Systems \& Allied Technologies, pp. 99-102, 30 August-1 September 2000, University of Brighton, UK.
[7] Dairoku Sekiguchi, Masahiko Inami and Susumu Tachi, RobotPHONE:RUI for Interpersonal Communication, CHI2001 Extended Abstracts, pp.277-278, 2001

[8] Noriyoshi SHIMIZU, Naoya KOIZUMI, Maki SUGIMOTO, Hideaki NII, Dairoku SEKIGUCHI, Masahiko INAMI,Teddy-bear based Robotic User Interface, Proceedings of ACM SIGCHI International Conference on Advances in Computer Entertainment Technology ACE 2005, pp.75-82, Jun. 2005. 